\title{
Design and Development of a Compact Triple-Band Microstrip Patch Antenna Loaded with Shorting Pin.
}

\author{
Hrudya B Kurup, Remsha M, Sruthi Dinesh, Stephen Rodrigues
}

\begin{abstract}
A single layer coaxial probe fed compact shorted patch antenna capable of triple band operation for GSM, ISM and WiMax application has been designed, fabricated and analyzed. The designed antenna generates three separate resonances to cover $1.9 \mathrm{GHz}$ DCS, Bluetooth and WLAN (2.4 GHz), $3.5 \mathrm{GHz}$ WiMax bands while maintaining a small overall size of $36 * 28 * 1.6$ mm3. A prototype is fabricated and experimental results show good radiation characteristics over the operating bands.
\end{abstract}

Keywords: Compact antennas, Mobile antennas, Shorted patch antenna, Quarter-wave patch; Triple-band antenna;

\section{INTRODUCTION}

Due to the advancements in the wireless communication field, handheld devices has become an integral part of the day to day life. Antennas are crucial components that determine the performance of these wireless devices. This increasing demand for wireless connectivity makes it necessary for a single antenna to cover several allocated frequency bands, utilizing less space. One way to achieve this is by using antennas having wide bandwidth. But when wideband antennas are used additional band pass filters are required to avoid collision and interference with coexisting communication bands. Hence there is large demand for multiband antennas with small size that are capable of multi-band operation.

A reduction in the patch size of the antenna can be achieved by placing a short-circuiting plane fully or partially, in the structure, where the electric field of the resonant mode is zero [I, 2], or by deploying a short-pin near to the feed probe. [3] However it is reported that patch size can be significantly reduced by deploying a single shorting pin/post instead of the typically used short circuit plane [4].This paper

Revised Manuscript Received on December 30, 2019.

* Correspondence Author

Hrudya.B.Kurup*, Department of instrumentation, Cochin University of Science and Technology, Cochin , Kerala, India, hrudyabalachandran@gmail.com

Ramsha M, Department of electronics, Cochin University of Science and Technology, Cochin , Kerala, India, remsha398@gmail.com

Sruthi Dinesh, Research Scholar, Department of Electronics, Cochin University of Science and Technology, Cochin, India

Stephen Rodrigues, Department of instrumentation, Cochin University of Science and Technology, Cochin , Kerala, India, dr3stephen@yahoo.co.in

(C) The Authors. Published by Blue Eyes Intelligence Engineering and Sciences Publication (BEIESP). This is an open access article under the CC BY-NC-ND license (http://creativecommons.org/licenses/by-nc-nd/4.0/) discusses the design and development of a single shorting pin loaded microstrip patch antenna, having an overall size of $36 * 28 * 1.6 \mathrm{~mm}$ capable of multi-band operation.

\section{ANTENNA DESIGN}

The present antenna consist of a top radiating patch and a ground plane on either side of a dielectric substrate along with a coaxial feed to excite the structure. The top metallic radiating portion consist of a rectangular patch with a rectangular ring circumscribing it. The rectangular patch is designed initially using the well-known equations available. $[5,6]$. Then a thin decagonal ring and a cross slot are inscribed in the patch for getting the desired resonance. To optimize the bandwidth a parasitic concentric square ring is placed.

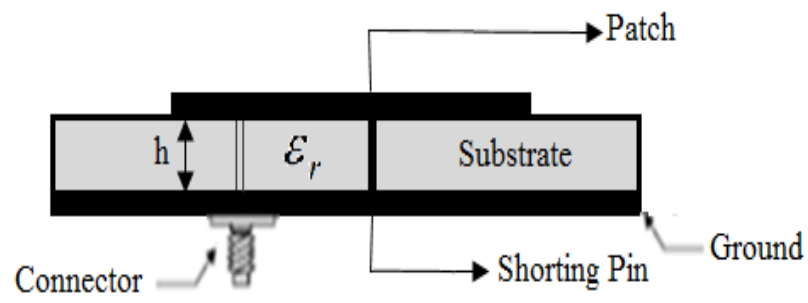

Fig. 1.Figure. 1.Cross Sectional view .

A short connecting the ground plane and top radiating patch is also present in the structure. The short in the structure is realized using a metallic pin which is inserted through a drilled hole connecting the patch and the ground plane in the substrate [5]. By using a shorting pin in the microstrip antenna, patch size can be reduced as well as multi-frequency operation, change of polarization etc. can be achieved [7, 8]. The schematic side view of the proposed antenna with a probe-feed is shown in fig.1.

\section{A. Basic features and working}

To comprehend the working of the antenna, the structure can be equivalently considered of as a slot and short loaded rectangular patch antenna. When loaded with shorting pin and slots the effective electrical length of the patch gets modified and hence tunable or multi frequency antenna is realized. The resonance frequency of the rectangular microstrip patch using a single shorting pin positioned in the middle of the width of the patch can be approximately calculated using the equation 


$$
f_{0}=\frac{3}{4\left(L_{e}+W_{e} / 2\right) \sqrt{\epsilon_{e}}} \mathrm{GHz}
$$

But this formula neglects the effect of the shorting post diameter which plays a significant role in the radiation characteristics of the antenna. [9] The slots present in the structure increases the effective dimensions of the upper radiating conductor per unit area of the surface, which leads to increase in both the equivalent inductance and capacitance of the structure. By introducing the short the antenna becomes quarter wavelength in its operation, thus reducing the half wavelength patch size, of a conventional microstrip patch antenna. The shorting pin in the structure can be modelled as an inductance, parallel to the equivalent resonant LC circuit of the existing patch. As a result new resonance modes are created. The parasitic rectangular ring which is placed around the shorted rectangular patch on the same substrate helps in optimizing the desired bandwidth. [10] Thus by the use of optimal slots in the radiating patch and by incorporating a single shorting pin, compact operation can be achieved.

The antenna is designed using FR-4 as dielectric material having a substrate thickness, $\mathrm{h}=1.6 \mathrm{~mm}$. The loss tangent and dielectric constant of FR-4 are respectively $\delta=0.02$ and $\varepsilon_{\mathrm{r}}=3.65$. The antenna structure is excited using a coaxial feed. The length and width of the patch are $22 * 17 \mathrm{~mm}^{2}$ whereas the ground plane dimensions are $28 * 36 \mathrm{~mm}^{2}$. The shorting pin used in this structure has a radius of $0.6 \mathrm{~mm}$. The top view of the metallic patch is shown in fig. 2 .

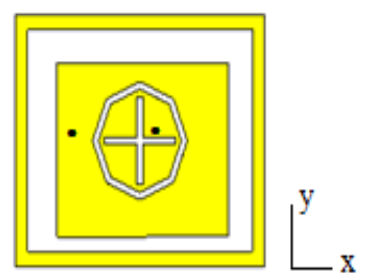

Figure. 2. Top view of the patch.

\section{B. Parametric Study}

A parametric study of the present antenna is conducted using CST Microwave studio, which yields worthwhile insight into the working of the radiator. The parametric study is basically concentrated towards the effect of shorting pin parameters like its position with respect to feed, the radius of the pin and height of the pin. Initially patch with and without short is compared and the results are shown in the table I.

\begin{tabular}{|c|c|}
\hline Antenna Structure & $\begin{array}{c}\text { Resonant Frequency } \\
(\mathrm{GHz})\end{array}$ \\
\hline With shorting pin & $\mathrm{f} 1=1.96$ \\
\cline { 2 - 2 } & $\mathrm{f} 2=2.44$ \\
\cline { 2 - 2 } & $\mathrm{f} 3=3.74$ \\
\hline Without short pin & $\mathrm{f} 1=2.44$ \\
\cline { 2 - 2 } & $\mathrm{f} 2=3.75$ \\
\hline
\end{tabular}

It can be noted that by introducing the shorting pin a new lower resonance is created, which is in accordance with the greens function-based theory of shorting posts. [11] By adjusting the pin position with respect to the feed position frequency tuning and enough impedance matching can be achieved.

To study the effect of shorting pin position, its location is varied, lengthwise (x) and widthwise (y) while keeping the feed position fixed. It can be seen that the minimum impedance mismatch points, changes significantly while varying the position of the shorting pin along the $\mathrm{x}$ axis, with a notable effect on the lower frequency which was introduced by the shorting pin. However while varying it along the $y$ axis the variation is minimum for all the three frequencies. This effect can be attributed to the effect of reactance (inductive and capacitive effect) introduced by the shorting pin with respect to the feed pin position. When the pin is positioned near to the feed, a strong capacitive reactance induces between the feeding pin and the short due to field coupling whereas when the short is placed away from the feed pin, the coupling of field reduces and the reactance offered by the pin becomes more inductive. This effect becomes more predominant as the distance between the pin increases. Thus shift in shorting pin position induces a shift in the zero reactance point of the antenna.

Since the feed is along $\mathrm{x}$ axis in this structure, the short pin position variation along the $\mathrm{x}$ axis changes the distance between the feed and the short significantly whereas along $y$ axis the distance variation is less. This explains the resonant frequency variation in the graph according to the position of the short with respect to the feed pin position along $\mathrm{x}$ and $\mathrm{y}$ axis. The graphs indicating the variation of frequencies with respect to the short position along $\mathrm{x}$ and $\mathrm{y}$ axis are shown in fig.3.

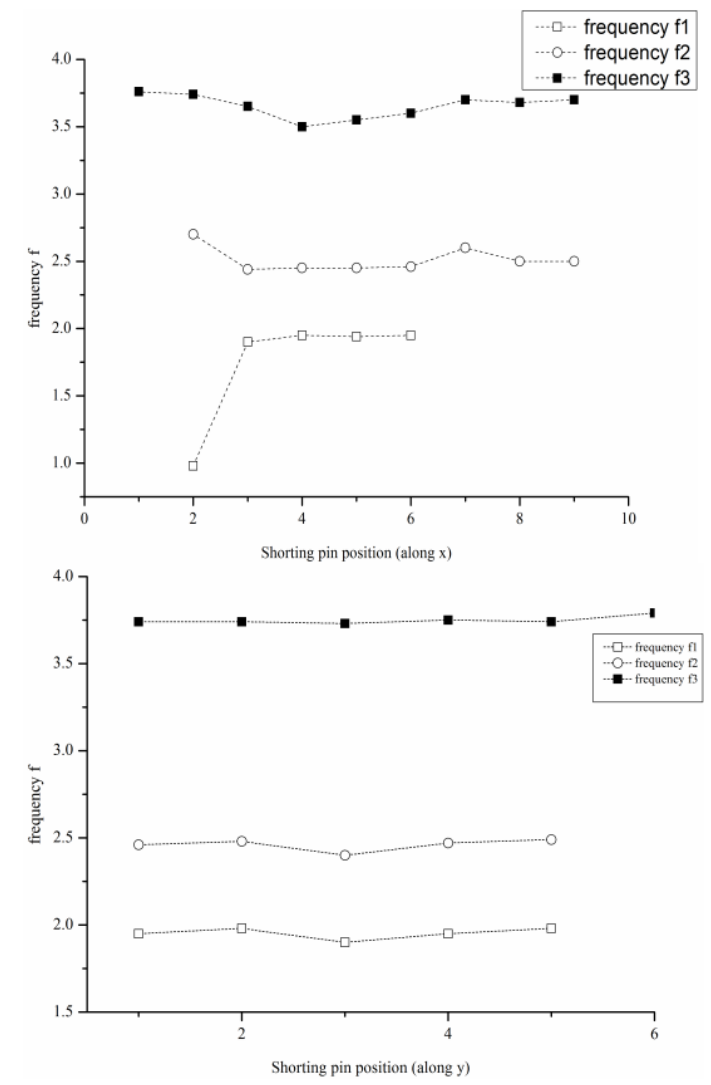

Figure. 3. Variation of resonant frequencies for different shorting pin positions.

ublished By: 
However within a fixed distance from the feed point the above mentioned change is minimal which can be used to fine-tune the frequencies for optimum operation.

The radius of the short is changed, keeping the other parameters fixed and the corresponding variation in resonant frequencies is studied. The graph in fig. 4 shows the variation in resonant frequencies with respect to the change in radius of the short. When the radius of the shorting pin increases the first resonance is relocated to a higher frequency range as the effective quarter-wavelength becomes smaller. However the second resonance has only a small variation whereas the third resonant frequency has little change when the radius of the pin is increased. Matching of the antenna is improved as the shorting pin radius is increased from 0.2 to $1.2 \mathrm{~mm}$.

The substrate thickness is varied to obtain the required Q-factor and bandwidth. It is noted that by simply increasing the substrate height the bandwidth or matching does not increase significantly, but give rise to distortions in impedance characteristics due to surface wave excitations. Studies were conducted for substrate heights of $0.8,1.6$ and $3.2 \mathrm{~mm}$. Optimum results are obtained for substrate height of $1.6 \mathrm{~mm}$. However when an air gap is introduced between the top patch and the ground plane and is tuned for proper matching, bandwidth increases at the expense of increased volume.

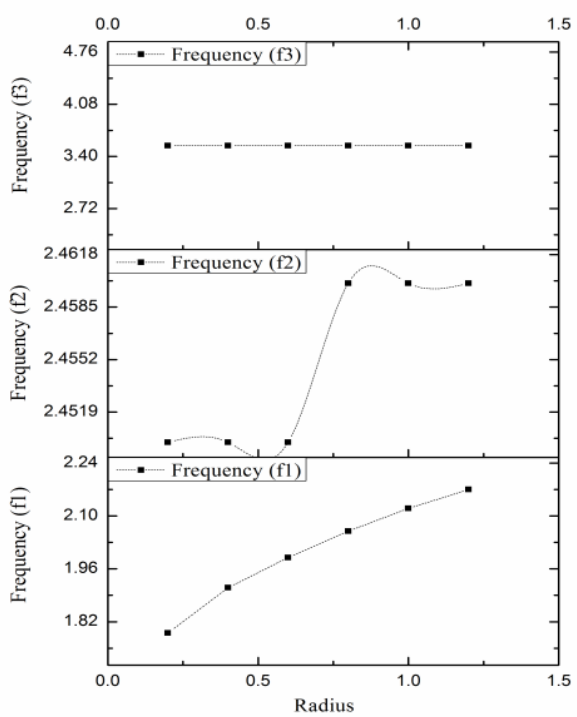

\section{Figure. 4. Variation of resonant frequencies for different shorting pin radius.}

The study demonstrates how the various shorting pin parameters affect the radiation characteristics of the antenna. It is clear from the study that, the effect of the short in the structure depends on various parameters like the number of pins, position of pins with respect to feed, the radius pin and the thickness of the substrate which in turn determines the length of the pin.

\section{FABRICATION}

A preliminary version of the proposed antenna is designed, constructed and measured. The antenna is etched on to a $1.6 \mathrm{~mm}$ thick FR-4 substrate and is short circuited using a thin copper wire of about $1.2 \mathrm{~mm}$ diameter. A very narrow hole was drilled in the substrate material between metallic patch and ground plane for inserting the shorting pin. One end of the shorting pin is then soldered with the patch and the other end to the ground plane. The structure is then fed using a coaxial feed. The photograph of the antenna prototype are shown in fig. 5.

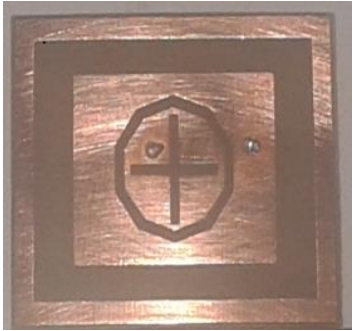

Figure. 5. The prototype of the antenna.

\section{RESULTS AND DISCUSSION}

The fabricated antenna is tested using a measurement system consisting of a network analyzer and antenna positioner in an anechoic chamber. The performance of the system is then evaluated by comparing results of proposed system with simulation results.The reflection coefficient variation with frequency, when simulated as well as measured, are as shown in fig 6.

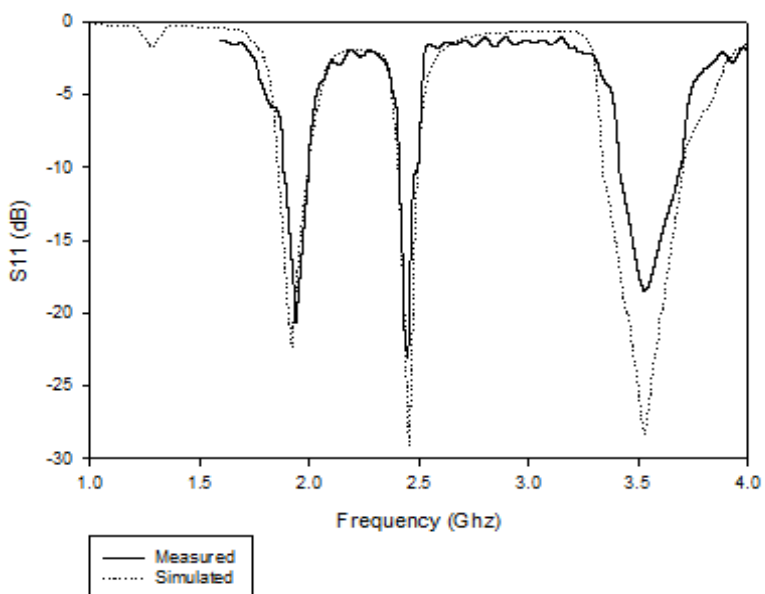

Figure. 6. Measured and simulated return loss plot.

The S11 (reflection coefficient) of the fabricated antenna structure is studied with the help of a vector network analyzer. As shown in fig.6, the simulated resonant frequencies of the antenna are obtained at $1.93 \mathrm{GHz}, 2.46$ $\mathrm{GHz}$ and 3.53. The measured result of antenna provides first resonant frequency at $1.95 \mathrm{GHz}$, second resonant frequency at $2.44 \mathrm{GHz}$ and third at and $3.52 \mathrm{GHz}$ respectively. The slight variation between the simulated and measured results are due to fabrication tolerance and material losses.

The simulated radiation pattern in all the three bands is a nearly Omni-directional one, with maximum radiation in broad side direction that gets slightly tapered towards the ground portion of the antenna. The patterns were also measured in anechoic chamber and are as shown in Figure 7. 

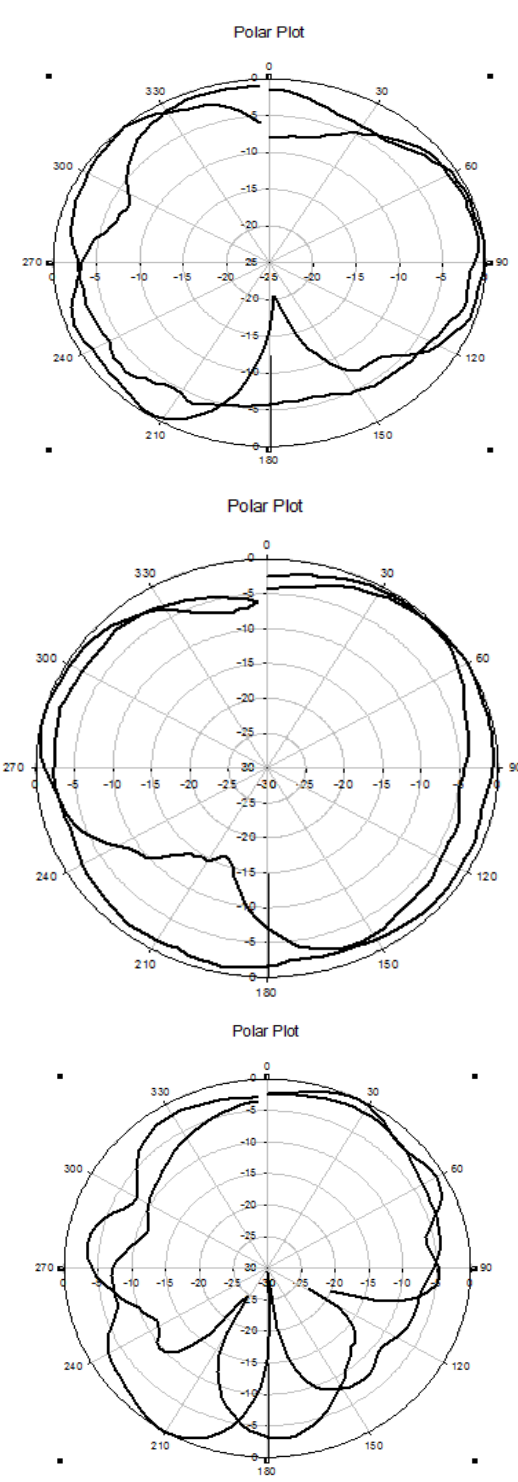

Figure. 7. Measured radiation patterns of proposed antenna in $E$ plane and $H$ plane at 1.9, 2.45 and 3.5 GHz.

The patterns are similar to a conventional shorted patch having a small ground-plane and the measured radiation pattern of the antenna is in agreement with the simulated results. The average antenna gain measured across all the three frequency bands is $1.5 \mathrm{dBi}$ with a maximum gain variation of $0.25 \mathrm{~dB}$.

\section{CONCLUSION}

The antenna presented in this paper is compact, low-cost and low-profile which is appropriate for DCS, Bluetooth, WLAN and WiMax Bands. The advantage of this type of antenna when compared to microstrip antenna is that it is resonant at a quarter-wavelength, resulting in reduced space utilization. By exciting the structure at a suitable feed position, and by strategically placing the shorting pin, good impedance matching condition for all the three resonant frequencies are obtained that makes it suitable for multi-band operation using a single coaxial feed. Parametric study shows that characteristics of the short such as its position, radius, and distance from the feed are crucial parameters that affect the working of the antenna. The antenna has omnidirectional radiation pattern in the horizontal plane and is suitable for hand held devices that need multiband capability and compact size.

\section{REFERENCES}

1. K. Hirasawa, and M. Haneishi (Eds.), "Analysis, design, and measurement of small and low-profile antennas", Artech House pp.73-74, 1992

2. S. Pinhas, and S. Shtrikman, "Comparison between computed and measured bandwidth of quarter-wave miccrostrip radiators", ZEEE Trans., vol. AP-36, no. 11, pp. 1615-1616,1988

3. R.B. Waterhouse, "Small microstrip patch antenna", Electron. Lett., vol 3 1, no. 8, pp.604-605,1995

4. Waterhouse, R.B., Targonski, S.D., and Kokotoff, D.M.: 'Design and performance of small printed antennas', IEEE Trans., 1998,AP-46, pp. 1629-1633.

5. Ramesh Garg, Prakash Bhartia, Inder Bahal and Apisak Ittipibon., "Microstrip Antenna Design Handbook," Artech House. Norwood MA Chapter 8, 2001.

6. Balanis C., "Antenna Theory: Analysis and Design". Third Edition, Wiley. New Jersey. (2005).

7. K. L. Wong and W. S. Chen, "Compact microstrip antenna with dual-frequency operation," Electronics Letters, Vol. 33, No. 8, pp. 646-647, 1997.

8. A. Vallecchi, G. B. Gentili, and M. Calamia, "Dual-band dual polarization microstrip antenna," Proceedings, IEEE International Symposium, pp. 134-137, 2003.

9. Girish Kumar and K.P. Ray, "Broadband microstrip antennas", Artech House antennas and propagation library, ISBN 1-58053-244-6, 2003

10. D.M. Kokotoff, R.B. Waterhouse, J.T. Aberle. An annular ring coupled to a shorted patch. IEEE Trans. Antennas Propagat. , 913 - 914.

11. R. Porath, "Theory of miniaturized shorting-post microstrip antennas," IEEE Transactions, Antennas and Propagation, Vol. 48, No. 1, pp. 41-47, 2000- 676

\section{ACKNOWLEDGMENT}

Acknowledging Council of Scientific \& Industrial Research (CSIR), India for their support during the work.

\section{AUTHORS PROFILE}

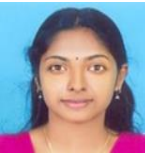

Hrudya.B.Kurup Research Scholar, Department of Instrumentation, Cochin University of Science and Technology, Cochin, India

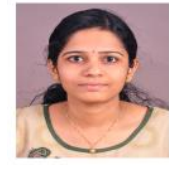

Remsha M Research Scholar, Department of Electronics, Cochin University of Science and Technology, Cochin, India.

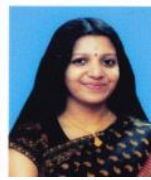

Sruthi Dinesh Research Scholar, Department of Electronics, Cochin University of Science and Technology, Cochin, India

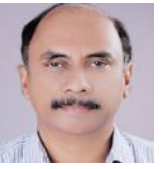

Stephen Rodrigues Professor, Department of Instrumentation, Cochin University of Science and Technology, Cochin, India 